\title{
Robust Estimation for Brain Tumor Segmentation
}

\author{
Marcel Prastawa ${ }^{1}$, Elizabeth Bullitt ${ }^{2}$, Sean $\mathrm{Ho}^{1}$, and Guido Gerig ${ }^{1,3}$ \\ ${ }^{1}$ Dept. of Computer Science, \\ ${ }^{2}$ Dept. of Surgery, \\ ${ }^{3}$ Dept. of Psychiatry \\ University of North Carolina, Chapel Hill, NC 27599, USA \\ prastawa@cs.unc.edu
}

\begin{abstract}
Given models for healthy brains, tumor segmentation can be seen as a process of detecting abnormalities or outliers that are present with certain image intensity and geometric properties. In this paper, we propose a method that segments brain tumor and edema in two stages. We first detect intensity outliers using robust estimation of the location and dispersion of the normal brain tissue intensity clusters. We then apply geometric and spatial constraints to the detected abnormalities or outliers. Previously published tumor segmentation methods generally rely on the intensity enhancement in the T1-weighted image that appear with the gadolinium contrast agent, on strictly uniform intensity patterns and most often on user initialization of the segmentation. To our knowledge, none of the methods integrated the detection of edema in addition to tumor as a combined approach, although knowledge of the extent of edema is critical for planning and treatment. Our method relies on the information provided by the (non-enhancing) T1 and T2 image channels, the use of a registered probabilistic brain atlas as a spatial prior, and the use of a shape prior for the tumor/edema region. The result is an efficient, automatic segmentation method that defines both, tumor and edema.
\end{abstract}

\section{Introduction}

Automatic brain tumor segmentation from MR images is a challenging task that offers exposure to various disciplines covering pathology, MRI physics, radiologist's perception, and image analysis based on intensity and shape . Recently, it has been shown that blood vessels in the brain exhibit certain characteristics within the pathological regions [1]. An objective and reproducible segmentation procedure coupled with vascular analysis would allow us to study the relation between pathologies and blood vessels and may function as a new diagnostic measure.

Previous work on brain tumor segmentation typically uses the enhancement provided by the gadolinium contrast agent in the T1 channel or blobby shaped tumors with uniform intensity [2,3]. Even though the intensity enhancement can aid the segmentation process, we show that it is not always necessary to obtain 

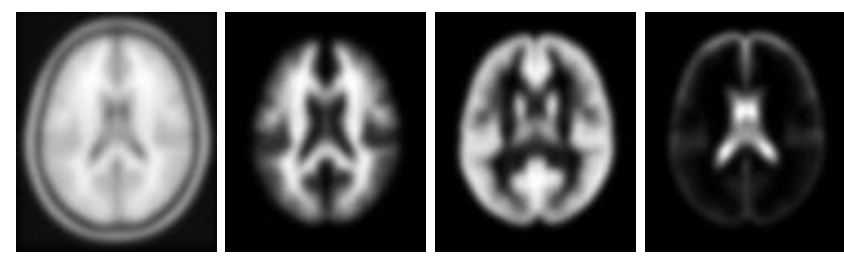

Fig. 1. The ICBM digital brain atlas. From left to right: the T1 template image and probability values of white matter, gray matter, and csf.

good results. In fact, the use of a contrast agent can be problematic. Typically, tumors are only partially enhanced and some early developing tumors are not enhanced at all. Blood vessels also generally appear enhanced by the contrast agent. These inconsistencies create an ambiguity in the image interpretation, which makes the T1-enhanced image channel a less than ideal feature for tumor segmentation.

Edema surrounding tumors and infiltrating mostly white matter was most often not considered as important for tumor segmentation. We showed previously [3] that edema can be segmented using a prior for edema intensity and restriction to the white matter region. The extraction of the edema region is essential for diagnosis, therapy planning, and surgery. It is also essential for attempts that model brain deformation due to tumor growth. The swelling produced by infiltrating edema most likely has distinctly different tissue property characteristics than tumor. Our new scheme presented here is based on the detection of "changes from normal" and will thus systematically include segmentation of edema. Differential identification of the two abnormal regions tumor and edema is clinically highly relevant. Even though the primary therapeutic focus will be on the tumor region, the edema region may require secondary analysis and treatment.

Our method combines the model of the normal tissues and the geometric and spatial model of tumor and edema. It relies only on the information provided in the T1 and T2 image channels. Tumor and edema are treated as intensity abnormalities or outliers. After identifying the abnormalities, an unsupervised clustering technique is applied to the intensity features before utilizing geometric and spatial constraints. We will demonstrate that this method can segment tumors with or without intensity enhancements and automatically detects the presence of edema, thus overcoming limitations of our previous method [3]. Our approach offers a means of approaching lesions of multiple types and image intensities, and, with a single method, lesions that enhance and do not, and that may or may not be surrounded by edema.

\section{Density Estimation and Detection of Abnormalities}

Manual brain tumor segmentation, the current gold standard, is a time consuming and tedious process that involves identifying image regions in $3-\mathrm{D}$ volumes that deviate from the expected intensities. Considering this perspective, we can 


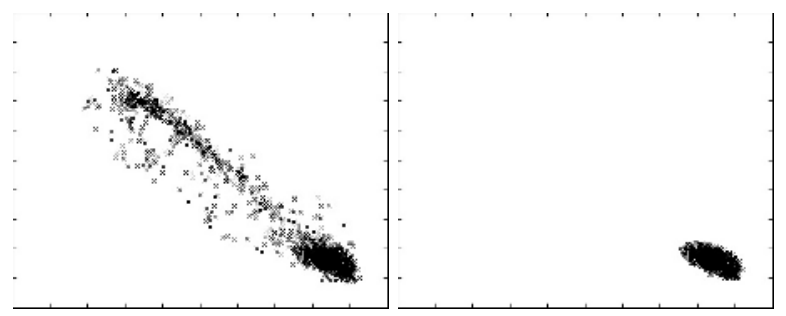

Fig. 2. The white matter training data for a subject with tumor and edema, the horizontal axis represents the T1 intensities and the vertical axis represents the T2 intensities. Left: original samples obtained by atlas-guided sampling which is contaminated with samples from other distributions. Right: remaining samples after trimming using the robust MCD estimate.

treat the process of segmenting tumors as the process of identifying intensity and spatial outliers. Gering et al. [4] proposed a method that relies on the detection of abnormalities. Their method is an extension of the Expectation-Maximization technique that uses information contained in multiple layers. These information layers involve voxel intensities, spatial coherence, structural relationships, and user input.

The initial densities for the normal brain tissues is obtained using a probabilistic brain atlas shown in Fig. [1 [5]. The image data is registered using affine transformation with an atlas template using the mutual information criterion [6. Training samples are obtained by taking a random subset of the voxels with high probability values. The set of samples is constrained to be the voxels with probabilities higher than $85 \%$ of the maximum probability for each class. The training data usually contains unwanted samples due to contamination with samples from other tissue types, especially tumor and edema. Our approach is to treat these contaminants as outliers and remove them from the training data.

The training samples for the normal brain tissue classes (white matter, gray matter, and cerebrospinal fluid/csf) are constrained to be well clustered. To impose this constraint, we use the robust estimate of the location and dispersion of the samples and remove samples that are not close to the location estimate (Fig. 2). The robust estimate used is the one given by the MCD (Minimum Covariances Determinant) estimator. It is defined to be the ellipsoid that covers at least half of the data with the lowest determinant of covariance. A fast algorithm for computing the MCD estimate is described in 7]. Cocosco et al. [8] used an alternative approach by trimming the training samples using MST (Minimum Spanning Trees) and edge breaking.

Given the trimmed training samples for the normal brain tissue classes, we estimate the probability density functions for each class. This allows us to find voxels with abnormal intensities, where we define abnormal regions to be the ones with low posterior probabilities of white matter, gray matter, and csf within the brain.

Tumors do not always appear with uniform intensities, particularly in the case where some tissues inside the tumor are necrotic tissues. We therefore make 
no assumption regarding the intensity distributions and use a non-parametric model for the probability density functions. Each density is approximated using kernel expansion or Parzen windowing [9]. The density function for the class label $\Gamma_{j}$ is $p\left(x \mid \Gamma_{j}\right)=\frac{1}{N} \sum_{i=1}^{N} K_{\lambda}\left(x-t_{i}\right)$ where $K$ is the multivariate Gaussian kernel with standard deviation $\lambda$ and $t_{i}$ is a class training sample. The kernel bandwidth $\lambda$ is set to be $4 \%$ of the data range.

At this stage, the goal is to compute the density estimates and posterior probabilities for the class labels $\Gamma=\{$ white matter, gray matter, csf, abnormal, non-brain $\}$. The iterative steps involved in fitting the density estimates to the image data are as follows:

1. Compute the values of the spatial priors using the atlas probabilities and initialize the density functions using atlas-guided sampling.

2. Compute the posterior probabilities.

3. Estimate bias field from white matter mask and apply correction.

4. Threshold the posterior probabilities and sample the high confidence regions. Trim the samples for normal tissues using the MCD estimate.

5. Estimate the non-parametric density for each class labels using kernel expansions.

6. Repeat steps 2 to 5 until the change in posterior probabilities is below a certain threshold or a maximum number of iterations is reached.

At the initialization step, the spatial priors are set to be the atlas probabilities for white matter, gray matter, and csf. For the outlier/abnormal class, we use a fraction of sum of the white matter and gray matter atlas probabilities since tumor and edema usually appear in these regions and not in the csf regions. The density functions for normal tissues is initialized using the trimmed samples after thresholding the atlas probabilities. The initial density for the abnormal class is set to be uniform, which makes this class act as a rejection class. The brain voxels with intensity features that are different from those of healthy classes or not located in the expected spatial coordinates will be assigned to this class.

The bias field correction is performed iteratively, in the spirit of the method described in [10]. In step 3, the bias field is estimated using the difference of the white matter voxels and the white matter mean, this field is constrained to be smooth. More sophisticated bias field correction techniques can be used in this step 1112. This iterative algorithm typically generates a good result after 5 iterations. The abnormal class density at different iterations for the Tumor020 data is shown in Fig. 3

\section{Extracting Tumor and Edema}

The densities and posterior probabilities computed for the abnormal class is a rough estimate of how likely the voxels are part of tumor or edema. Given this estimate, the next step would be to identify the tumor and edema voxels. We do this in two steps, the first is to separate the densities into two clusters and the second is to apply geometric and spatial constraints to the segmented structures. 

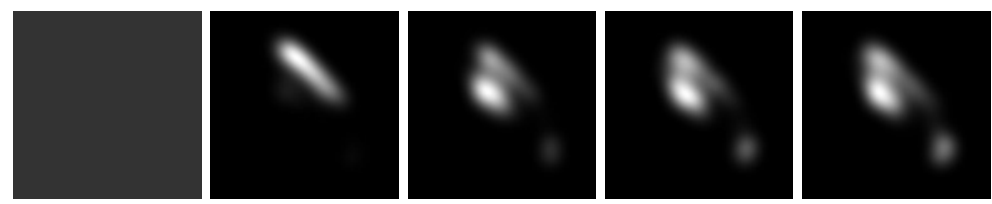

Fig. 3. The density of the abnormal class at different iterations of the fitting process for the Tumor020 data. The horizontal axis represents the T1 intensities and the vertical axis represents the $\mathrm{T} 2$ intensities. The two high density regions visible at the final iteration are the tumor and edema densities, which have a significant separation along the dimension of the T2 intensities.

\subsection{Cluster Separation}

Tumor and edema are generally separable given the information in the T2 channel. Since edema has high fluid content it appears brighter than tumor in this modality. To separate the densities, we sample the regions with high probability of abnormality and apply unsupervised clustering to generate the training data for tumor and edema. The method we have chosen is k-means clustering with $k=29$. Once we obtain the clusters, we can identify the tumor cluster as the cluster with the T2 mean that has the lower value. The tumor and edema density estimates are then obtained using kernel expansion.

Edema does not necessarily appear together with tumor, therefore we should only perform the cluster separation if there exists a strong evidence. For a measure of validity of the separation, we use the overlap measure called the DaviesBouldin index [13]. This measure is the ratio of the average within cluster distances and the between cluster distance. The T2 channel contains most of the information needed for differentiating tumor and edema. Therefore, we have chosen to measure the overlap for only the T2 data of each cluster. If the amount of overlap is larger than a specified threshold, then the tumor density is set to be the density for the abnormal class and the edema density is set to zero.

\subsection{Geometric and Spatial Constraints}

We assume that tumor structures appear as blobby lumps and that edema structures are connected to the tumor structures. Here, we use the prior knowledge that edema, if present, is always contiguous with the tumor. The shape constraint is enforced by applying a region competition snakes with the tumor probability as the input [14]. The spatial constraint is enforced by setting edema probabilities that are not connected to a tumor region to zero.

Once we obtain the initial tumor and edema densities and posterior probabilities, we then fit the densities to the image data using the iterative steps described in section 2. The fitting algorithm for this stage is modified with the addition of an extra step where these geometric and spatial constraints are enforced. The set of class labels used at this stage is $\Gamma=\{$ white matter, gray matter, csf, tumor, edema, non-brain\}. The tumor shape constraint is disabled at the last fitting stage. This is done to obtain the proper boundary for the tumor 
$\mathrm{T} 1$
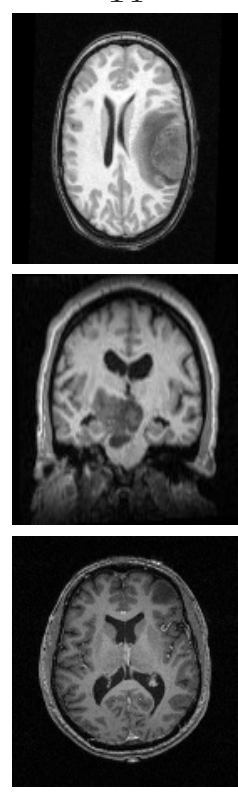

$\mathrm{T} 2$
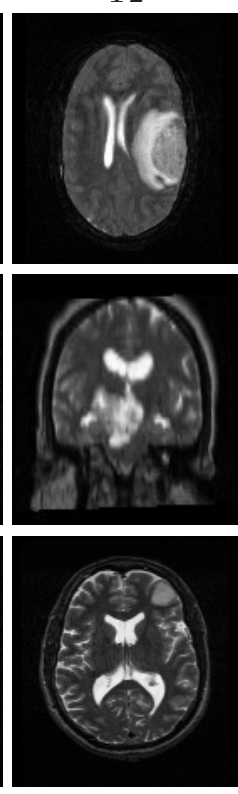

Tumor
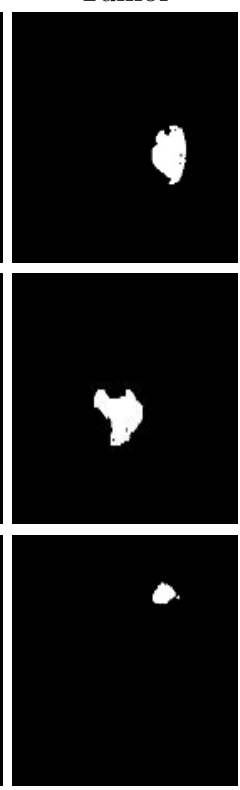

Edema
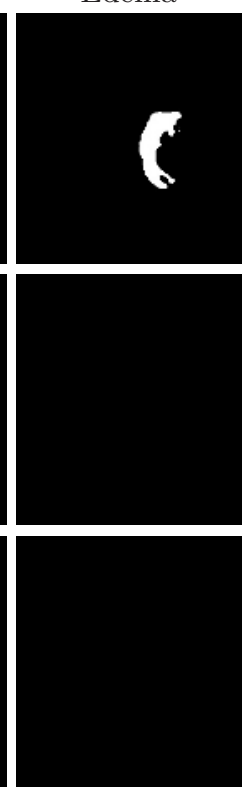

3D View
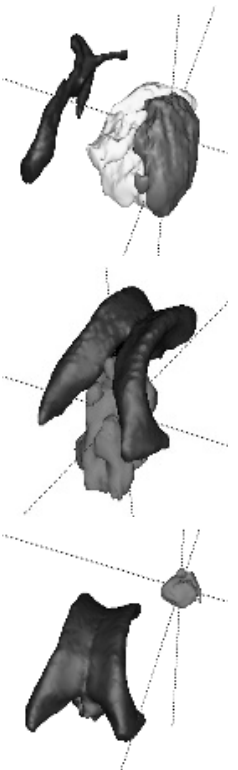

Fig. 4. The datasets and the generated segmentation results. The last column shows the $3 \mathrm{D}$ views of the segmented structures: medium gray represents tumor, bright gray represents edema, and dark gray represents ventricles. From top to bottom: Tumor020, Tumor025, Tumor033. These results illustrate that our method does differential segmentation for tumor and edema, which works also in cases where no edema is present.

structures, which may not be entirely smooth. For instance, gliomas generally have ragged boundaries.

\section{Results}

We have applied the method to three real datasets as shown in Fig. 4. Tumor020 has a partially enhancing tumor that causes a large deformation of the normal structures. Tumor025 contains a large, partially enhancing tumor inside the brain stem. Tumor033 contains a low grade tumor which is not highlighted in the T1enhanced channel.

The automatically generated tumor mask is compared with hand segmentation results. We used the VALMET segmentation validation tool [15] to generate the five metrics shown in Fig. 5] The volume overlap measure is the normalized voxel intersection count for the pair of segmentations A and B: $(A \cap B) /(A \cup B)$, otherwise known as Jaccard's similarity coefficient [16]. The other measures are the maximum Hausdorff distance and the average surface distances (inside, outside, and absolute). 


\begin{tabular}{|c|c|r|r|r|r|r|}
\hline Dataset & Tissue Type & Overlap & Hausdorff & Inside & Outside & Absolute \\
\hline \hline Tumor020 & Tumor & $80.0 \%$ & 16.79 & 1.28 & 2.16 & 1.64 \\
\hline Tumor020 & Edema & $68.2 \%$ & 12.80 & 0.63 & 2.43 & 1.75 \\
\hline Tumor025 & Tumor & $79.2 \%$ & 17.85 & 1.01 & 3.70 & 1.44 \\
\hline Tumor033 & Tumor & $70.6 \%$ & 8.60 & 0.25 & 2.47 & 1.85 \\
\hline
\end{tabular}

Fig. 5. Validation metrics of the automatic tumor segmentation results against manual results. The surface distances are measured in voxels.

\section{Discussion and Conclusion}

This paper presents a new approach for automatic segmentation of tumors and adjoining edema from dual-channel MRI (T1 and T2 weighted channels). Alternative methods so far have either relied on mostly enhancing, homogeneous tumors. Further, they need user-guidance in training a supervised classifier or roughly outline the region of interest. Here, we show that robust estimation and outlier detection can be a promising new concept for detecting abnormalities in the brain. First shown in this paper, we present a technique that identifies tumor and edema, if present. Our collaborating clinicians confirm that this is a highly relevant feature, as the edema region often may require secondary analysis and treatment after the primary focus to the tumor region. The technique uses a concept that detects difference from normal and uses non-parametric estimates for distributions rather than traditional mixture Gaussian models. With the addition of prior knowledge of the shape of brain tumor and location of edema, automatic segmentation of tumor and edema is made possible.

Continuing research will couple this new concept with our previously developed Expectation-Maximization based tissue and tumor segmentation scheme [3], which showed good performance if the tumor was partially enhanced after contrast-injection. This paper shows three cases, with and without edema, processed with exactly the same scheme. We will apply the method to a set of over 15 archived routine tumor cases which all exhibit tumors which are highly variable in appearance, size, location, and structure. Validation will be possible since all these cases were also processed using a user-guided tool for tumor segmentation.

Acknowledgments. This work was supported, in part, by NIH-NIBIB R01 EB000219 and NIH-NCI R01 HL69808. Color version is available at http://www.cs.unc.edu/〜prastawa/papers/MICCAI2003_RobustTumor.pdf

\section{References}

1. Bullitt, E., Gerig, G., Pizer, S.M., Aylward, S.R.: Measuring tortuosity of the intracerebral vasculature from MRA images. IEEE Transactions on Medical Imaging (2003) in print, available at http://casilab.med.unc.edu. 
2. Kaus, M.R., Warfield, S.K., Nabavi, A., Chatzidakis, E., Black, P.M., A., J.F., R., K.: Segmentation of meningiomas and low grade gliomas in MRI. In Taylor, C., Colchester, A., eds.: MICCAI. Volume 1679 of LNCS., Springer (1999) 1-10

3. Moon, N., Bullitt, E., Van Leemput, K., Gerig, G.: Automatic brain and tumor segmentation. In Dohi, T., Kikinis, R., eds.: Medical Image Computing and Computer-Assisted Intervention MICCAI 2002. Volume 2489 of LNCS., Springer Verlag (2002) 372-379

4. Gering, D.T., Grimson, W.E.L., Kikinis, R.: Recognizing deviations from normalcy for brain tumor segmentation. In Dohi, T., Kikinis, R., eds.: Medical Image Computing and Computer-Assisted Intervention MICCAI 2002. Volume 2488 of LNCS., Springer Verlag (2002) 388-395

5. Evans, A.C., Collins, D.L., Mills, S.R., Brown, E.D., Kelly, R.L., Peters, T.M.: 3D statistical neuroanatomical models from 305 MRI volumes. In: Proc. IEEE Nuclear Science Symposium and Medical Imaging Conference. (1993) 1813-1817

6. Maes, F., Collignon, A., Vandermeulen, D., Marchal, G., Suetens, P.: Multimodality image registration by maximization of mutual information. IEEE Transactions on Medical Imaging 16 (1997) 187-198

7. Rosseauw, P.J., Van Drissen, K.: A fast algorithm for the minimum covariance determinant estimator. Technometrics 41 (1999) 212-223

8. Cocosco, C.A., Zijdenbos, A.P., Evans, A.C.: Automatic generation of training data for brain tissue classification from mri. In Dohi, T., Kikinis, R., eds.: Medical Image Computing and Computer-Assisted Intervention MICCAI 2002. Volume 2488 of LNCS., Springer Verlag (2002) 516-523

9. Duda, R.O., Hart, P.E., Stork, D.: Pattern Classification. second edn. Wiley (2001)

10. Wells, W.M., Kikinis, R., Grimson, W.E.L., Jolesz, F.: Adaptive segmentation of MRI data. IEEE Transactions on Medical Imaging 15 (1996) 429-442

11. Van Leemput, K., Maes, F., Vandermeulen, D., Suetens, P.: Automated modelbased bias field correction of MR images of the brain. IEEE Transactions on Medical Imaging 18 (1999) 885-896

12. Shattuck, D.W., Sandor-Leahy, S.R., Schaper, K.A., Rottenberg, D.A., Leahy, R.M.: Magnetic resonance image tissue classification using a partial volume model. NeuroImage 13 (2001) 856-876

13. Davies, D.L., Bouldin, D.W.: A cluster separation measure. IEEE Transactions on Pattern Analysis and Machine Intelligence 1 (1979) 224-227

14. Ho, S., Bullitt, E., Gerig, G.: Level set evolution with region competition: Automatic 3-D segmentation of brain tumors. In Katsuri, R., Laurendeau, D., Suen, C., eds.: Proc. 16th International Conference on Pattern Recognition, IEEE Computer Society (2002) 532-535

15. Gerig, G., Jomier, M., Chakos, M.: VALMET: a new validation tool for assessing and improving 3D object segmentation. In Niessen, W., Viergever, M., eds.: Medical Image Computing and Computer-Assisted Intervention MICCAI 2001. Volume 2208., New York, Springer Verlag (2001) 516-523

16. Jaccard, P.: The distribution of flora in the alpine zone. New Phytologist 11 (1912) $37-50$ 